\title{
Some properties of Reinhardt and Hartogs domains
}

LUDMILA BOURCHTEIN

ANDREI BOURCHTEIN

\begin{abstract}
The domains of certain types, such as Reinhardt and Hartogs, are used in different problems of theory of functions of several complex variables. For instance, any power series of several complex variables converges in the complete logarithmically convex Reinhardt domain. Transformations of Reinhardt and Hartogs allow us to diminish the dimension of space and to consider some type of domains using its images. This allows the visibility of the geometric representation and the simplification of the study of properties of such domains. In this article we consider some properties of Reinhardt and Hartogs domains and transformations. The properties of domain conservation under Reinhardt transformation and convexity conservation under Reinhardt and Hartogs transformation are proved.
\end{abstract}




\section{RESUMO}

Os domínios de certos tipos, tais como os de Reinhardt e Hartogs, são usados em diferentes problemas de teoria de funções de várias variáveis complexas. Por exemplo, qualquer série de potências de várias variáveis complexas converge num domínio completo logaritmicamente convexo de Reinhardt. Transformações de Reinhardt e Hartogs permitem diminuir a dimensão do espaço e considerar alguns tipos de domínios utilizando sua imagem. Isto possibilita visualizar a representação geométrica e simplificar o estudo de propriedades desses domínios. Neste artigo consideremos algumas propriedades de domínios e transformações de Reinhardt e de Hartogs. Demonstraremos a propriedade de conservação do domínio sob transformação de Reinhardt e a de conservação de convexidade sob transformações de Reinhardt e de Hartogs.

Keywords: several complex variables, Reinhardt domain, Hartogs domain, convexity

\section{INTRODUCTION. COMPLEX SPACE $C^{n}$ AND SOME SIMPLE DOMAINS}

Reinhardt and Hartogs domains were introduced at the beginning of the 20th century in order to solve some problems arisen in the theory of functions of several complex variables, in particular, to find the domain of convergence of the power and Hartogs series [4]. As it is well known, the domain of convergence of the power series is a complete Reinhardt domain $[2,4]$.

Convexity and connected concept of pseudoconvexity are important properties for characterization of domains of convergence. For example, not every complete Reinhardt domain is the precise domain of convergence of some power series in the case of the several complex variables. The holomorphic convexity, introduced by Cartan and Thullen, serves for global characterization of domains of holomorphy and has a central place in the modern theory of several complex variables [2,4]. Therefore, an investigation 8 Ciência \& Natura, Santa Maria, 24: 7 - 20, 2002. 
of the convexity properties of Reinhardt and Hartogs domains is important problem. In this paper we apply Reinhardt and Hartogs transformations $[4,6]$ to simplify this investigation and to provide geometric representation of these domains.

Let us introduce some basic definitions.

The complex $n$-dimensional space $C^{n}$ is the set of complex-valued vectors (points) with $n$ components

$$
z=\left(z_{1}, z_{2}, \ldots, z_{n}\right)=\left\{z_{k}\right\}_{k=1}^{n} \text {. }
$$

Since $z_{k}=x_{k}+i y_{k}=x_{k}+i x_{n+k}$, the points of $C^{n}$ are the points of real $2 n$-dimensional space $R^{2 n}$. In the space $C^{n}$ the vector space structure over the complex field $C$ can be introduced, that is, the rules for vector addition and multiplication by complex numbers can be defined as follows [1]:

$$
\begin{gathered}
z+w=\left(z_{1}+w_{1}, z_{2}+w_{2}, \ldots, z_{n}+w_{n}\right), \forall z, w \in C^{n} ; \\
\lambda z=\left(\lambda z_{1}, \lambda z_{2}, \ldots, \lambda z_{n}\right), \forall z \in C^{n}, \forall \lambda \in C .
\end{gathered}
$$

The Euclidean norm is introduced by formula $[1,3]$

$$
|z|^{2}=\sum_{k=1}^{n}\left|z_{k}\right|^{2}
$$

The Euclidean norm $|z|$ coincides with norm of vector $z$ in the space $R^{2 n}$. Also, polydisc norm can be introduced $[3,6]$ :

$$
\|z\|=\sup _{k=1 . n}\left|z_{k}\right|
$$

These two norms are connected by the following inequalities $[3,6]$ :

$$
\|z\| \leq|z| \leq n^{1 / 2}\|z\| \text {. }
$$

Let us consider some simple domains in the space $C^{n}$ (hereinafter domain is an open connected set).

1. The disc with center $a \in C^{n}$ and radius $r$ is

$$
B(a, r)=\left\{z \in C^{n}:|z-a|<r\right\}
$$

This is Euclidean disc in the space $R^{2 n}$, its boundary is $(2 n-1)$ dimensional circle

$$
\partial B(a, r)=\left\{z \in C^{n}:|z-a|=r\right\}
$$


2. Polydisc with center $a \in C^{n}$ and radius $r \in R$ is

$$
U(a, r)=\left\{z \in C^{n}:\|z-a\|<r\right\}
$$

Polydisc is the disc with center $a$ in polydisc metric and it represents the Cartesian product of $n$ plane discs with centers $a_{k}$ and radius $r$. There exists generalized polydisc with center $a=\left(a_{1}, a_{2}, \ldots, a_{n}\right) \in C^{n}$ and vector radius $r=\left(r_{1}, r_{2}, \ldots, r_{n}\right) \in R^{n}$ :

$$
U(a, r)=\left\{z \in C^{n}:\left|z_{k}-a_{k}\right|<r_{k}, k=1, \ldots, n\right\}
$$

3. A Reinhardt domain with center $a \in C^{n}$ satisfies the following property: if point $z^{0}=\left\{z_{k}^{0}\right\}_{k=1}^{n}$ belongs to this domain then all points in the form $z=\left\{a_{k}+\left(z_{k}^{0}-a_{k}\right) \exp \left(i \theta_{k}\right)\right\}_{k=1}^{n}, 0 \leq \theta_{k}<2 \pi$, belong to this domain, too $[3,6]$.

A Reinhardt domain with center $a \in C^{n}$ is called complete if together with any point $z^{0}$ it contains all points $z=\left\{z_{k}\right\}_{k=1}^{n} \quad$ which satisfy inequality: $\quad\left|z_{k}-a_{k}\right| \leq\left|z_{k}^{0}-a_{k}\right|$, $\forall k=1, \ldots, n$. Otherwise, Reinhardt domain is incomplete $[3,6]$.

Obviously, disc and polydisc are complete Reinhardt domains. If $n=1$ then complete Reinhardt domains are discs $\{|z-a|<R\}$ and incomplete Reinhardt domains are circular rings $\{r<|z-a|<R\}$.

Without loss of generality we can consider the Reinhardt domains with center in the origin $(a=0)$. In this case, if a point $z=\left\{z_{k}\right\}_{k=1}^{n}$ belongs to a such domain, then the same happen to all points with the same modulus of components $\left|z_{k}\right|, k=1, \ldots, n$ and any argument.

Let us consider Reinhardt transformation [6]

$$
z \rightarrow \alpha(z)=\left(\left|z_{1}\right|,\left|z_{2}\right|, \ldots,\left|z_{n}\right|\right)=\left\{\left|z_{k}\right|\right\}_{k=1}^{n}
$$

from complex $n$-dimensional space $C^{n}$ into real $n$-dimensional space $R^{n}$, more precisely onto $R_{+}^{n}=R_{+} \times . \times R_{+}$, where 
$R_{+}=[0 ;+\infty)$. This Reinhardt transformation $\alpha: C^{n} \rightarrow R_{+}^{n}$ maps a Reinhardt domain $D$ into set of points $D_{+} \subset R_{+}^{n}$ which is called the Reinhardt image (or diagram) of the domain $D$. If $D$ is a complete Reinhardt domain then the set $D_{+}$contains together with any point $\left\{\left|z_{k}^{0}\right|\right\}_{k=1}^{n}$ all rectangular parallelepiped $\left\{\left|z_{k}\right| \leq\left|z_{k}^{0}\right|, \forall k=1, \ldots, n\right\}$. This diagram characterizes completely the Reinhardt domains and makes possible their geometric representations in the cases $n=2$ and $n=3$.

4. A Hartogs domain with symmetry plane $z_{n}=a_{n}$ satisfies the following property: if a point $z^{0}=\left\{z_{k}^{0}\right\}_{k=1}^{n}$ belongs to this domain then all points of the form $z=\left(z_{1}^{0}, z_{2}^{0}, \ldots, z_{n-1}^{0}, a_{n}+\left(z_{n}^{0}-a_{n}\right) \exp \left(i \theta_{n}\right)\right), 0 \leq \theta_{n}<2 \pi$ belong to this domain too [6].

A Hartogs domain is called complete if for each element $z^{0}$ it contains all points $z=\left\{z_{k}\right\}_{k=1}^{\prime \prime}$ which satisfy the constraints: $z_{k}=z_{k}^{0}, k=1, \ldots, n-1,\left|z_{n}-a_{n}\right| \leq\left|z_{n}^{0}-a_{n}\right|$ [6]. Obviously, the set of Hartogs domains is wider than the set of Reinhardt domains.

Let us consider the Hartogs domains with symmetry plane $z_{n}=0$ and introduce the Hartogs transformation $\beta: C^{n} \rightarrow C^{n-1} \times R_{+}$by formula [6]

$$
z \rightarrow \beta(z)=\left(z_{1}, z_{2}, \ldots, z_{n-1},\left|z_{n}\right|\right)
$$

We denote by ' $z=\left(z_{1}, \ldots, z_{n-1}\right)$ the projection of the point $z \in C^{n}$ into space $C^{n-1}$ and by ' $D$ the projection of the domain $D$ into space $C^{n-1}$ (that is, the set of all projections ' $z$ of the points $z \in D$ ). The image of a complete Hartogs domain contains together with any point $\left(z^{0},\left|z_{n}^{0}\right|\right)$ the entire segment $\left\{\left(z^{0},\left|z_{n}\right|\right):\left|z_{n}\right| \leq\left|z_{n}^{0}\right|\right\}$. Hartogs diagram reduces dimension in 1 and makes possible geometric representation of Hartogs domains if $n=2$. 


\section{CONSERVATION OF DOMAIN UNDER REINHARDT TRANSFORMATION}

Theorem 1. Reinhardt transformation (6) maps any domain $D \subset C^{n}$, which does not intersect a set $E=\left\{z_{1} \cdot z_{2} \cdot \ldots z_{n}=0\right\}$, onto a domain of the space $R^{n}$.

Proof. Let us denote by $G$ the image of a domain $D$ under Reinhardt transformation (6): $G=\alpha(D)$, where $\alpha(z)=\left\{\left|z_{k}\right|\right\}_{k=1}^{n}=\left\{\alpha_{k}\right\}_{k=1}^{n}$.

1) First, we prove that set $G$ is open, that is, all of its points are interior points. Let $\alpha^{0}=\left(\alpha_{1}^{0}, \alpha_{2}^{0}, \ldots, \alpha_{n}^{0}\right)$ be an arbitrary point of set $G$. Because $G$ is image of domain $D$ under transformation $\alpha(z)$, then there exists $z^{0} \in D, \quad z^{0}=\left(z_{1}^{0}, \ldots, z_{n}^{0}\right)$ such that $\alpha\left(z^{0}\right)=\alpha^{0}$. Then,

$$
\alpha^{0}=\left(\alpha_{1}^{0}, \alpha_{2}^{0}, \ldots, \alpha_{n}^{0}\right)=\left(\left|z_{1}^{0}\right|,\left|z_{2}^{0}\right|, \ldots,\left|z_{n}^{0}\right|\right) \text {. }
$$

Since the domain $D$ does not intersect the set $E=\left\{z_{1} \cdot z_{2} \cdot \ldots \cdot z_{n}=0\right\}$, then $\left|z_{k}^{0}\right| \neq 0, \forall k=1, \ldots, n$. Hence, we can rewrite the point $z^{0} \in D$ in the form $z^{0}=\left(z_{1}^{0}, \ldots, z_{n}^{0}\right)=\left(\left|z_{1}^{0}\right| \exp \left(i \varphi_{1}^{0}\right), \ldots,\left|z_{n}^{0}\right| \exp \left(i \varphi_{n}^{0}\right)\right)$. Since $D$ is a domain, there exists a radius $r>0$ such that the disc with center $z^{0}$ and radius $r$ is contained in $D: B\left(z^{0}, r\right)=\left\{\left|z-z^{0}\right|<r\right\} \subset D$. We now consider the disc $B\left(\alpha^{0}, r\right)=\left\{\left|\alpha-\alpha^{0}\right|<r\right\}$ in the set $R_{+}^{n}$. If we choose any point $\widetilde{\alpha}=\left(\widetilde{\alpha}_{1}, \ldots, \widetilde{\alpha}_{n}\right) \in B\left(\alpha^{0}, r\right)$ and consider the point $\tilde{z}=\left(\tilde{\alpha}_{1} \exp \left(i \varphi_{1}^{0}\right), \ldots, \tilde{\alpha}_{n} \exp \left(i \varphi_{n}^{0}\right)\right) \in C^{n}$, then $\tilde{\alpha}=\alpha(\tilde{z})$ and the Euclidean distance between points $\tilde{z}$ and $z^{0}$ can be evaluated as follows:

$\left|\tilde{z}-z^{0}\right|=\left(\sum_{k=1}^{n}|| \tilde{z}_{k}\left|\exp \left(i \varphi_{k}^{0}\right)-\right| z_{k}^{0 \mid}\left|\exp \left(\left.i \varphi_{k}^{(0)}\right|^{2}\right)^{1 / 2}=\left(\sum_{k=1}^{n}\left(\left|\tilde{z}_{k}\right|-\left|z_{k}^{0}\right|\right)^{2}\right)^{1 / 2}=\right| \tilde{\alpha}-\alpha^{0} \mid<r\right.$ Therefore, $\tilde{z} \in B\left(z^{0}, r\right) \subset D$ (in particular, it follows from here that 
$\left.\left|\widetilde{z}_{k}\right|=\widetilde{\alpha}_{k} \neq 0, \forall k=1, \ldots, n\right)$ and, consequently, $\tilde{\alpha}=\alpha(\tilde{z}) \in G$. Thus, $B\left(\alpha^{0}, r\right) \subset G$, that is, $\alpha^{0}$ is an interior point of set $G$. Since $\alpha^{0}$ is an arbitrary point of set $G$, then set $G$ is open.

2) To prove that $G$ is connected set, we refer to wellknown result that the image of a connected set under a continuous mapping is connnected [5]. Since a Reinhardt transformation (6) is a continuous mapping of a metric space $C^{n}$ into a metric space $R^{n}$, $G=\alpha(D)$ is a connected set.

Thus, $G$ is open and connected, that is, $G$ is a domain.

$$
\text { Observation. Condition } D \cap E=\varnothing, E=\left\{z_{1} \cdot z_{2} \cdot \ldots \cdot z_{n}=0\right\}
$$
is important. In fact, the image $G$ of the bidisc $|z|<1$ ( $z=\left(z_{1}, z_{2}\right) \in C^{2}$ ) under the transformation $\alpha(z)$ is not a domain because it contains the parts $T_{1}=\left\{0 \leq\left|z_{1}\right|=\alpha_{1}<1,\left|z_{2}\right|=\alpha_{2}=0\right\}$ and $T_{2}=\left\{\left|z_{1}\right|=\alpha_{1}=0,0 \leq\left|z_{2}\right|=\alpha_{2}<1\right\}$ of its boundary (this is shown in Fig.1).

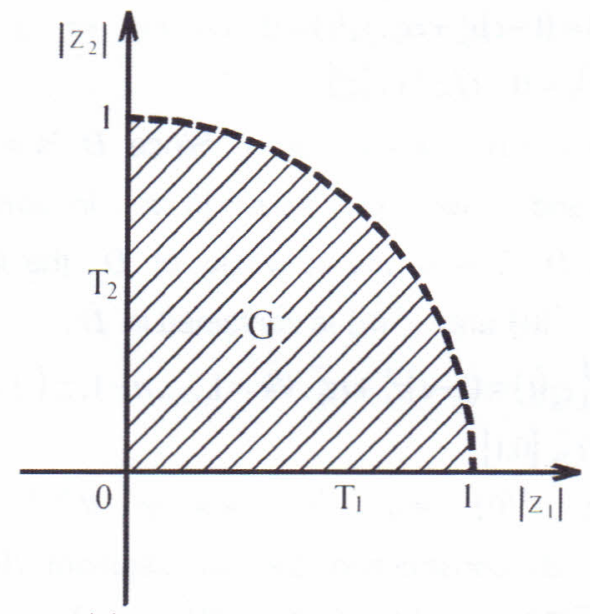

Fig. 1. Image of bidisc $|z|<1$ under Reinhardt transformation. The parts $T_{1}$ and $T_{2}$ of boundary belong to image. 


\section{CONVEXITY OF HARTOGS DOMAIN}

Theorem 2. A complete Hartogs domain $D \subset C^{n}$ is convex if, and only if, its image $\beta(D)$ under Hartogs transformation (7) is a convex set in the space $R^{2 n-1}$.

Proof. First, we prove the necessary condition. Let $D$ be a complete and convex Hartogs domain. We must prove that its image $G=\beta(D)$ under Hartogs transformation (7) is a convex set in the space $R^{2 n-1}$. Let $\beta^{1}=\left(\beta_{1}^{1}, \beta_{2}^{1}, \ldots, \beta_{n}^{1}\right)$ and $\beta^{2}=\left(\beta_{1}^{2}, \beta_{2}^{2}, \ldots, \beta_{n}^{2}\right)$ be two arbitrary points of the set $G$. The points $\beta^{1}$ and $\beta^{2}$ are images of some points $z^{1}(\varphi)=\left(z_{1}^{1}, \ldots, z_{n}^{1}\right)=\left(z_{1}^{1}, \ldots, z_{n-1}^{1},\left|z_{n}^{1}\right| \exp (i \varphi)\right) \quad$ and $z^{2}(\psi)=\left(z_{1}^{2}, \ldots, z_{n}^{2}\right)=\left(z_{1}^{2}, \ldots, z_{n-1}^{2},\left|z_{n}^{2}\right| \exp (i \psi)\right)$ under Hartogs transformation, that is $\beta^{1}=\left(\beta_{1}^{1}, \ldots, \beta_{n-1}^{1}, \beta_{n}^{1}\right)=\left(z_{1}^{1}, \ldots, z_{n-1}^{1},\left|z_{n}^{1}\right|\right)$ and $\beta^{2}=\left(\beta_{1}^{2}, \ldots, \beta_{n-1}^{2}, \beta_{n}^{2}\right)=\left(z_{1}^{2}, \ldots, z_{n-1}^{2},\left|z_{n}^{2}\right|\right)$. The line segment joining the points $\beta^{1}$ and $\beta^{2}$ in the space $R^{2 n-1}$ has the following representation:

$$
\beta(t)=\left\{\begin{array}{l}
x_{k}(t)=(1-t) x_{k}^{1}+t x_{k}^{2}, y_{k}(t)=(1-t) y_{k}^{1}+t y_{k}^{2}, \forall k=\overline{1, n-1}, \\
\left|z_{n}(t)\right|=(1-t)\left|z_{n}^{1}\right|+t\left|z_{n}^{2}\right|
\end{array}\right\}, 0 \leq t \leq 1,
$$

where $z_{k}=x_{k}+i y_{k}, \forall k=1, \ldots, n-1$. Since $D$ is a Hartogs domain, $z^{1}(\varphi) \in D$ and $z^{2}(\psi) \in D, \forall \varphi, \psi \in[0 ; 2 \pi]$, in particular, $z^{1}(0) \in D$ and $z^{2}(0) \in D$. Due to the convexity of $D$, the line segment $l(t)$ connecting $z^{1}(0)$ and $z^{2}(0)$ is contained in $D$ :

$l(t)=z(t)=\left\{z_{k}(t)=(1-t) z_{k}^{1}+t z_{k}^{2}, \forall k=1, \ldots, n-1, z_{n}(t)=(1-t)\left|z_{n}^{1}\right|+t\left|z_{n}^{2}\right|\right\}$ , $z(t) \in D, \forall t \in[0 ; 1]$.

The points $z^{1}(0)$ and $z^{2}(0)$ are in $R^{2 n-1}$ and $z^{1}(0)=\beta^{1}$, $z^{2}(0)=\beta^{2}$. By construction, the line segment $l(t)$ coincides with $\beta(t)$. From $\quad l(t) \in D, \forall t \in[0 ; 1], \quad$ it follows that $\beta(t)=\beta(l(t)) \in G, \forall t \in[0 ; 1]$, that is, the line segment $\beta(t)$ lies entirely in $G$. Therefore $G$ is a convex set. 
Now we prove the sufficient condition. Let $D$ be a complete Hartogs domain and its image $G=\beta(D)$ under Hartogs transformation be a convex set. We show that $D$ is convex. Let $z^{1}=\left(z_{1}^{1}, \ldots, z_{n}^{1}\right)$ and $z^{2}=\left(z_{1}^{2}, \ldots, z_{n}^{2}\right)$ be two arbitrary points of the domain $D$. The line segment

$$
l(t)=z(t)=\left\{z_{k}(t)\right\}_{k=1}^{n}=\left\{(1-t) z_{k}^{1}+t z_{k}^{2}\right\}_{k=1}^{n}, 0 \leq t \leq 1
$$

joins these points. Transformation $\beta(z)$ maps $z^{1}, z^{2}$ and $l(t)$ onto points $\beta^{1}=\beta\left(z^{1}\right)=\left(z_{1}^{1}, \ldots, z_{n-1}^{1},\left|z_{n}^{1}\right|\right), \quad \beta^{2}=\beta\left(z^{2}\right)=\left(z_{1}^{2}, \ldots, z_{n-1}^{2},\left|z_{n}^{2}\right|\right)$ and curve

$$
\lambda(t)=\beta(l(t))=\left\{z_{k}(t)=(1-t) z_{k}^{1}+t z_{k}^{2}, \forall k=1, \ldots n-1,\left|z_{n}(t)\right|=\left|(1-t) z_{n}^{1}+t z_{n}^{2}\right|\right\}
$$

respectively. We can construct a line segment $\tilde{\lambda}(t)$ connecting the point $\beta^{1}$ and $\beta^{2}$ :

$\tilde{\lambda}(t)=\left\{z_{k}(t)=(1-t) z_{k}^{1}+t z_{k}^{2}, \forall k=1, \ldots, n-1,\left|\tilde{z}_{n}(t)\right|=(1-t)\left|z_{n}^{1}\right|+t\left|z_{n}^{2}\right|\right\}$

Since the points $\beta^{1}$ and $\beta^{2}$ belong to the set $G$, it follows from its convexity that $G$ contains the entire line segment $\tilde{\lambda}(t)$. Because

$$
\left|z_{n}(t)\right|=\left|(1-t) z_{n}^{1}+t z_{n}^{2}\right| \leq(1-t)\left|z_{n}^{1}\right|+t\left|z_{n}^{2}\right|=\left|\widetilde{z}_{n}(t)\right|
$$

and $D$ is a complete Hartogs domain, then all points of the curve $\lambda(t)$ belong to the set $G$. Therefore the preimage of the curve $\lambda(t)$, that is, the line segment $l(t)$ is contained in domain $D$. This signifies that $D$ is a convex domain.

\section{CONVEXITY OF REINHARDT DOMAIN}

Theorem 3. A complete Reinhardt domain $D \subset C^{n}$ is convex in $C^{n}$ if, and only if, its image $G=\alpha(D)$ under Reinhardt transformation (6) is a convex set in $R^{n}$.

Proof. First, we show the necessary condition. Let $D$ be a complete convex Reinhardt domain in $C^{n}$ and $G=\alpha(D)$ be the image of the domain $D$ under Reinhardt transformation 
$z \rightarrow \alpha(z)=\left(\left|z_{1}\right|, \ldots,\left|z_{n}\right|\right)$. The line segment $l(t)$ joining two arbitrary points $\alpha^{1}=\left(\alpha_{1}^{1}, \ldots, \alpha_{n}^{1}\right)$ and $\alpha^{2}=\left(\alpha_{1}^{2}, \ldots, \alpha_{n}^{2}\right)$ of set $G$ has the form

$$
l(t)=\alpha(t)=\left\{(1-t) \alpha_{k}^{1}+t \alpha_{k}^{2}\right\}_{k=1}^{n}, 0 \leq t \leq 1 .
$$

Since $\alpha^{1}, \alpha^{2} \in G$ and $G=\alpha(D)$, there exists two points $z^{1}=\left(z_{1}^{1}, \ldots, z_{n}^{1}\right)$ and $z^{2}=\left(z_{1}^{2}, \ldots, z_{n}^{2}\right)$ of the domain $D$ such that $\alpha^{1}=\alpha\left(z^{1}\right), \alpha^{2}=\alpha\left(z^{2}\right)$, that is,

$$
\alpha^{1}=\left(\alpha_{1}^{1}, \ldots, \alpha_{n}^{1}\right)=\left(\left|z_{1}^{1}\right|, \ldots,\left|z_{n}^{1}\right|\right), \alpha^{2}=\left(\alpha_{1}^{2}, \ldots, \alpha_{n}^{2}\right)=\left(\left|z_{1}^{2}\right|, \ldots,\left|z_{n}^{2}\right|\right) .
$$

From the definition of Reinhardt domains, $D$ contains the points that can be expressed by

$$
\begin{gathered}
z^{1}(\varphi)=z^{1}\left(\varphi_{1}^{1}, \ldots, \varphi_{n}^{\prime}\right)=\left(\left|z_{1}^{\prime}\right| \exp \left(i \varphi_{1}^{\prime}\right), \ldots,\left|z_{n}^{\prime}\right| \exp \left(i \varphi_{n}^{\prime}\right)\right), \\
0 \leq \varphi_{k}^{\prime}<2 \pi, \forall k=1, \ldots, n
\end{gathered}
$$

and

$$
\begin{aligned}
z^{2}(\varphi)=z^{2}\left(\varphi_{1}^{2}, \ldots, \varphi_{n}^{2}\right) & =\left(\left|z_{1}^{2}\right| \exp \left(i \varphi_{1}^{2}\right), \ldots,\left|z_{n}^{2}\right| \exp \left(i \varphi_{n}^{2}\right)\right), \\
& \leq \varphi_{k}^{2}<2 \pi, \forall k=1, \ldots, n .
\end{aligned}
$$

In particular, the points $z^{1}(0)$ and $z^{2}(0)$ belong to $D$ : $z^{1}(0)=\left(\left|z_{1}^{1}\right|, \ldots,\left|z_{n}^{1}\right|\right) \in D, \quad z^{2}(0)=\left(\left|z_{1}^{2}\right|, \ldots,\left|z_{n}^{2}\right|\right) \in D$. By noting that $z^{1}(0)=\alpha^{1}, z^{2}(0)=\alpha^{2}$, we conclude that the line segment

$$
l(t)=\alpha(t)=\left\{(1-t) \alpha_{k}^{1}+t \alpha_{k}^{2}\right\}_{k=1}^{n}=\left\{(1-t)\left|z_{k}^{1}\right|+t\left|z_{k}^{2}\right|\right\}_{k=1}^{n}
$$

joining the points $\alpha^{1}$ and $\alpha^{2}$ in Reinhardt diagram, at the same time connects the points $z^{1}(0)$ and $z^{2}(0)$ in domain $D$ and this line segment lies entirely in domain $D$ due to its convexity. Therefore the image $\alpha(l(t))=l(t)$ of the line segment $l(t)$ is contained in $G$, that is, the set $G$ is convex.

Now we show that Reinhardt diagram convexity implies the convexity of its preimage. Let $D$ be a complete Reinhardt domain and $G=\alpha(D)$ be a convex set, where $\alpha(z)$ is the Reinhardt transformation. The line segment $l(t)$ joining two arbitrary points $z^{1}=\left(z_{1}^{1}, \ldots, z_{n}^{1}\right)$ and $z^{2}=\left(z_{1}^{2}, \ldots, z_{n}^{2}\right), z^{1}, z^{2} \in D$ has the form: 


$$
l(t)=z(t)=\left\{(1-t) z_{k}^{1}+t z_{k}^{2}\right\}_{k=1}^{n}, 0 \leq t \leq 1 .
$$

Transformation $\alpha(z)$ maps $z^{1}, z^{2}$ and $l(t)$ onto points

$$
\alpha^{1}=\alpha\left(z^{1}\right)=\left\{\left|z_{k}^{1}\right|\right\}_{k=1}^{n}, \alpha^{2}=\alpha\left(z^{2}\right)=\left\{\left|z_{k}^{2}\right|\right\}_{k=1}^{n}
$$

and the curve

$$
\lambda(t)=\alpha(l(t))=\left\{\left|(1-t) z_{k}^{1}+t z_{k}^{2}\right|\right\}_{k=1}^{n}, 0 \leq t \leq 1,
$$

respectively. The curve $\lambda(t)$ joins the points $\alpha^{1}$ and $\alpha^{2}$ because $\lambda(0)=\alpha(l(0))=\left\{\left|z_{k}^{1}\right|\right\}_{k=1}^{n}$ and $\lambda(1)=\alpha(l(1))=\left\{\left|z_{k}^{2}\right|\right\}_{k=1}^{n}$. Since $\alpha^{1}, \alpha^{2} \in G$ and $G$ is convex, the line segment joining these two points

$$
\tilde{\lambda}(t)=\left\{(1-t)\left|z_{k}^{1}\right|+t\left|z_{k}^{2}\right|\right\}_{k=1}^{n}, 0 \leq t \leq 1
$$

is entirely contained in $G$. Obviously, the following inequalities hold for all $k=1, \ldots, n$

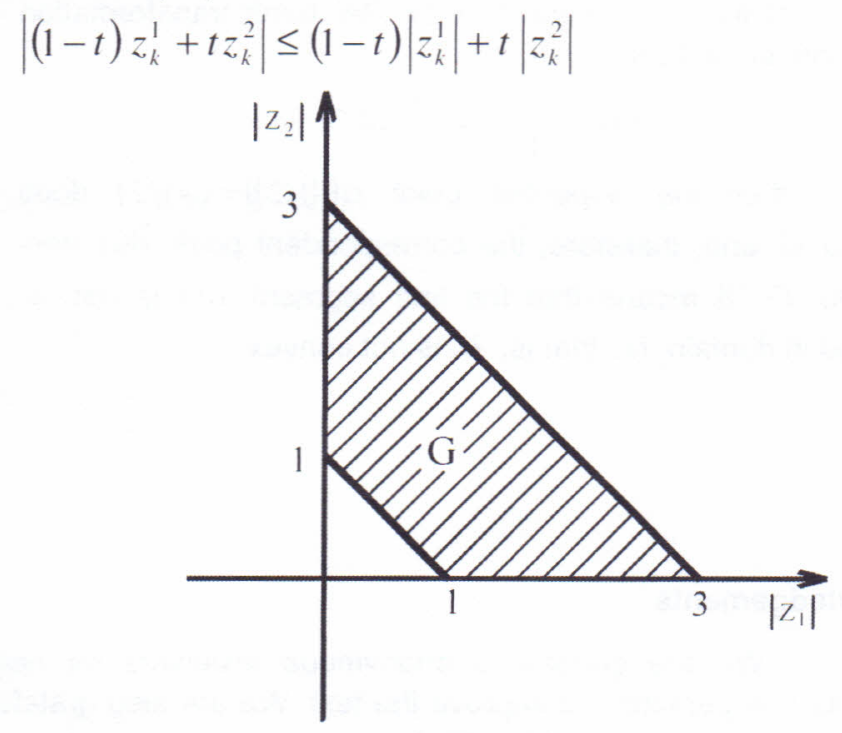

Fig. 2. Convex set representing Reinhardt diagram of domain defined by inequalities $1<\left|z_{1}\right|+\left|z_{2}\right|<3$. 
Since Reinhardt domain $D$ is complete, it follows from the condition $\tilde{\lambda}(t) \in G, \forall t \in[0 ; 1]$ and inequalities (8) that $\lambda(t) \subset G, \forall t \in[0 ; 1]$. Hence, $l(t) \subset D$, that is, $D$ is convex.

Observation. A completeness of the domain $D$ is important for convexity of both Reinhardt and Hartogs domains. In fact, the twice inequality $1<\left|z_{1}\right|+\left|z_{2}\right|<3$ defines a Reinhardt domain $D$ in the space $C^{2}$. The Reinhardt diagram $G=\alpha(D)$ of domain $D$ is a convex set in the plane $R^{2}$ (see Fig.2).

Let us consider the point $\alpha=(1 ; 1 / 2) \in G$. Since $D$ is a Reinhardt domain, then all the points $z(\varphi)=(\exp (i \varphi) ; 1 / 2), 0 \leq \varphi<2 \pi$ belong to $D$. We choose the points $z^{1}=z(0)=(1 ; 1 / 2) \in D$ and $z^{2}=z(\pi)=(-1 ; 1 / 2) \in D$ and join these ones with the line segment

$$
l(t)=\left((1-t) \cdot 1+t \cdot(-1),(1-t) \cdot \frac{1}{2}+t \cdot \frac{1}{2}\right)=\left(1-2 t ; \frac{1}{2}\right), 0 \leq t \leq 1 .
$$

The image of this line segment under Reinhardt transformation is a set of points in the form

$$
\alpha(l(t))=\left(|1-2 t|, \frac{1}{2}\right), 0 \leq t \leq 1 .
$$

If $t=1 / 2$, then the respective point $\alpha(l(1 / 2))=(0 ; 1 / 2)$ does not belong to $G$ and, therefore, the correspondent point $l(0)$ does not belong to $D$. It means that the line segment $l(t)$ is not entirely contained in domain $D$, that is, $D$ is not convex.

\section{Acknowledgements}

We are grateful to anonymous reviewers for helpful comments that permitted to improve the text. We are also grateful to the science foundation FAPERGS for supporting this work with a grant 01/60053.9. 


\section{References}

[1] H. Cartan, Elementary Theory of Analytic Functions of One or Several Complex Variables. New York, Dover Pub., 1995, 226p.

[2] L. Hormander, An Introduction to Complex Analysis in Several Variables. Amsterdam, North-Holland, 1990, 254p.

[3] R. Narasimhan, Several Complex Variables. Chicago, University of Chicago Press, 1995, 184p.

[4] M.R. Range, Holomorphic Functions and Integral Representations in Several Complex Variables. New York, Springer-Verlag, 1998, $388 p$.

[5] W. Rudin, Principles of Mathematical Analysis. New York, McGraw-Hill, 1976, 342p.

[6] B.V. Shabat, Introduction to Complex Analysis. Part 2: Functions of Several Variables. Providence, American Mathematical Society, 1992, 381p. 
LUDMILA BOURCHTEIN

ANDREI BOURCHTEIN

Department of Mathematics

Pelotas State University

Pelotas, RS - Brasil 